\title{
Sofosbuvir-Based Therapies Achieved Satisfactory Virological Response in Chinese Individuals with Genotypes 3 and 6 Infections: A Real-World Experience
}

\author{
Qiao Tang \\ Li Wei \\ Xiaoqing Liu \\ Peng $\mathrm{Hu}(\mathbb{D}$
}

Department of Infectious Diseases, Institute for Viral Hepatitis, The Key Laboratory of Molecular Biology for Infectious Diseases, Chinese Ministry of Education, The Second Affiliated Hospital of Chongqing Medical University, Chongqing, People's Republic of China
Correspondence: Peng Hu

Department of Infectious Diseases, Institute for Viral Hepatitis, The Key Laboratory of Molecular Biology for Infectious Diseases, Chinese Ministry of Education, The Second Affiliated Hospital of Chongqing Medical University, 74 Linjiang Road, Yuzhong District, Chongqing, 400000, People's Republic of China

Tel +86 I3608338064

Email hp_cq@163.com
Background: Previous studies have shown that sofosbuvir-based regimens yield high sustained virological response rates in patients with hepatitis $\mathrm{C}$ virus (HCV) infection except for genotype $3 \mathrm{~b}$ complicated with cirrhosis. This real-world study aims to explore the efficacy and safety of sofosbuvir-based regimens in Chinese patients with genotypes 3 and 6 infections, especially the impact of ribavirin coadministration on sustained virological response in cirrhotic patients with genotype $3 \mathrm{~b}$ infection.

Methods: This is a retrospective cohort study that included 101 patients initiated on sofosbuvir-based regimens. The main endpoint of treatment was sustained virological response at posttreatment week 12 (SVR12).

Results: Overall, the SVR12 rates were $95.0 \%$ (96/101); specifically, the rates were $100 \%$ in sofosbuvir, $88.2 \%$ in sofosbuvir+ribavirin, $100 \%$ in sofosbuvir+daclatasvir, $100 \%$ in sofosbuvir+daclatasvir+ribavirin, $95.0 \%$ in sofosbuvir/velpatasvir, and $97.1 \%$ in sofosbuvir/velpatasvir+ribavirin $(\mathrm{p}=0.534)$. The SVR12 rates were comparable in patients infected with genotypes 3 and 6 (93.2\% versus 97.6\%, p=0.339). The SVR12 rate was $93.9 \%$ in cirrhotic patients (31/33). Among those infected with genotype 3, the SVR12 rate was 91.7\% (22/24); the rate was $95.0 \%$ in those with ribavirin coadministration regimens, which was numerically higher than the $75.0 \%$ in those without ribavirin. However, no statistical difference was found $(\mathrm{p}=0.312$ ). In total, five patients failed to achieve SVR12, including 3 patients with genotype $3 \mathrm{~b}$ infection treated with ribavirin coadministration regimens (one of them was cirrhotic), 1 cirrhotic patient with genotype $3 \mathrm{k}$ infection, and 1 noncirrhotic patient with genotype 6a infection. No severe adverse event occurred.

Conclusion: Real-world data show that sofosbuvir-based regimens are highly effective and safe for patients with HCV genotypes 3 and 6 infections.

Keywords: daclatasvir, hepatitis $\mathrm{C}$ virus, ribavirin, sofosbuvir, velpatasvir

\section{Introduction}

It has been estimated that hepatitis $\mathrm{C}$ virus (HCV) genotype 3 is the next most common genotype worldwide, second only to genotype 1 , and that it accounts for $30.1 \%$ of cases, or approximately 54.3 million patients. ${ }^{1}$ In East Asia, genotype 1 is still dominant, followed by genotype $6(16.3 \%)$, which is more common than genotype 3 $(10.4 \%)$, which is ranked fourth. ${ }^{1}$ In China, genotype $1 \mathrm{~b}$ and genotype $2 \mathrm{a}$ are the two main genotypes; they are more prevalent than genotypes 3 and 6 . The prevalence of 
genotype 3 and 6 infections is increased significantly in the population of South China, and these patients are younger than the patients infected with other genotypes. ${ }^{2-4}$ It is well known that $\mathrm{HCV}$ infection increases the risk of hepatocellular carcinoma (HCC), end-stage liver disease and hepaticrelated death, especially in patients with detectable serum HCV-RNA. ${ }^{5,6}$ Moreover, patients with genotype 3 and 6 infections are more closely associated with the occurrence of HCC than other genotypes. ${ }^{7-9}$

Previous studies have shown that sofosbuvir (SOF)-based treatment yields high sustained virological response (SVR) rates in patients with $\mathrm{HCV}$ infection. ${ }^{10-14}$ However, it is recognized that the population infected with genotype 3, especially subtype $3 \mathrm{~b}$ complicated with liver cirrhosis, is characterized by a lower SVR rate at the time of direct antiviral agent (DAA) therapy. ${ }^{15-17}$ There is a standing debate about whether the addition of ribavirin (RBV) has an impact on SVR in HCV-infected patients. ${ }^{18-20}$ Unfortunately, most data on the efficacy and safety of SOF-based therapy in patients infected with genotype 3 and 6 infections are from Europe. However, these data are limited in China, especially regarding regimens of SOF/velpatasvir (VEL) with or without RBV. ${ }^{19,21-23}$ Therefore, there is an urgent need for more real-world research from China to be conducted.

The present real-world study aims to explore the efficacy and safety of SOF \pm RBV, SOF+daclatasvir (DCV) $\pm \mathrm{RBV}$ and SOF/VEL $\pm \mathrm{RBV}$ regimens in Chinese patients with genotype 3 and 6 infections, especially regarding whether RBV coadministration has an impact on SVR in patients with subtype $3 \mathrm{~b}$ infection and cirrhosis.

\section{Method}

\section{Study Design and Participants Selection}

This is a retrospective cohort study consisting of a total of 101 patients initiated on SOF-based regimens in routine practice from the Second Affiliated Hospital of Chongqing Medical University between July 2017 and December 2020. Patients infected with genotypes 3 and 6 were initiated on 6 kinds of SOF-based regimens, including SOF, SOF+DCV, and SOF/VEL regimens with or without RBV for regular duration, according to the guidelines, the availability of DAAs, and the physician. The inclusion criteria for screening participants were being aged 18 years or older with hepatitis C virus infection regardless of hepatitis B virus (HBV) coinfection, cirrhosis status, and treatment experience. This study was approved by the Ethical Committee of the Second Affiliated Hospital of Chongqing Medical University.

\section{Data Collection}

The following information was collected at baseline: general demographic features (sex, age), viral-related data (HCV genotype and level of serum HCV-RNA), clinical laboratory examinations (level of aspartate aminotransferase (AST), alanine aminotransaminase (ALT), total bilirubin, total bile acid, albumin, hemoglobin, platelet count and AFP), assessment of cirrhosis status and comorbidity (diabetes, chronic renal diseases, fatty liver disease) and coinfection with HBV. Serum HCV-RNA was determined by Roche Cobas Ampliprep/Cobas TaqMan or Kehua. Liver cirrhosis was diagnosed by an APRI index over 2, an FIB-4 index over 3.25, liver stiffness over $14.6 \mathrm{kPa}$, or ultrasonography imaging and examination indications.

\section{Outcome}

The treatment efficacy was determined by the sustained virological response at posttreatment week 12 (SVR12), which was defined as the main endpoint of treatment and determined by the improvement of fibrosis evaluated by FIB-4 $(($ Age $\times$ AST) $/($ PLT $\times($ square root of ALT)), and APRI ((AST/ULN*100)/PLT). ${ }^{24,25}$ The clinical symptoms and adverse events were monitored during the treatment and follow-up for safety and tolerance assessment.

\section{Statistical Analysis}

The differences in the continuous variables between pretreatment and posttreatment were compared by paired t-tests. A chi-square test was performed to compare categorical data. Variables that were associated with liver fibrosis progression with a $\mathrm{p}$-value $<0.1$ were included in multivariate logistic regression models. Two-sided $p$ values were calculated for all tests. A p-value $<0.05$ was considered statistically significant. Data were analyzed via SPSS version 24.0 (IBM Corp., Armonk, NY, USA).

\section{Results}

\section{Baseline Characteristics}

A total of 101 patients infected with genotypes $3(n=59)$ and $6(\mathrm{n}=42)$ who received SOF-based regimens were enrolled at initiation, and their baseline demographic and clinical characteristics are shown in Table 1. A total of $32.7 \%$ of patients had cirrhosis, and $7.9 \%$ had experience with treatment. The percentage of patients who received the $\mathrm{SOF} \pm \mathrm{RBV}$, SOF $+\mathrm{DCV} \pm \mathrm{RBV}$ and $\mathrm{SOF} / \mathrm{VEL} \pm \mathrm{RBV}$ regimens for regular duration accounted for $27.7 \%, 18.8 \%$, and $53.5 \%$, respectively. There was no significant difference in age, sex, level 
Table I Baseline Characteristics of Patients Who Received Sofosbuvir-Based Regimens with or without RBV and Diverse Genotypes

\begin{tabular}{|c|c|c|c|c|c|c|c|}
\hline & $\begin{array}{l}\text { Total } \\
(n=101)\end{array}$ & $\begin{array}{l}\text { With RBV } \\
(n=66)\end{array}$ & $\begin{array}{l}\text { Without RBV } \\
(n=35)\end{array}$ & $P$ value & $\begin{array}{l}\text { Genotype } 3 \\
(n=59)\end{array}$ & $\begin{array}{l}\text { Genotype } 6 \\
(n=42)\end{array}$ & $P$ value \\
\hline Age (y) & $42.8(19-76)$ & $43.0(19-76)$ & $42.5(22-64)$ & 0.821 & $41.7(22-59)$ & $44.4(19-76)$ & 0.188 \\
\hline Sex (males/female) & $65 / 36$ & $47 / 19$ & $18 / 17$ & 0.048 & $39 / 20$ & $26 / 16$ & 0.664 \\
\hline ALT (U/L) & II 7.7 (17-492) & $121.2(17-326)$ & $110.4(22-492)$ & 0.580 & |29|.8 (29-269) & $111.3(17-492)$ & 0.576 \\
\hline AST (U/L) & $83.2(19-436)$ & $80.7(19-244)$ & $88.3(28-436)$ & 0.606 & $89(22-252)$ & $74.5(19-436)$ & 0.305 \\
\hline Total bilirubin (mmol/L) & $17(3.9-70.2)$ & $17.3(4.6-70.2)$ & $16.3(3.9-39.5)$ & 0.705 & $16.6(4.4-70.2)$ & $17.5(3.9-68.1)$ & 0.740 \\
\hline Total bile-acid (mmol/L) & $17.9(0.5-124.7)$ & $19.0(0.5-124.7)$ & $16.2(1.4-67.6)$ & 0.675 & $21.4(0.5-124.7)$ & $13.2(0.8-67.6)$ & 0.203 \\
\hline Albumin $(g / L)$ & $42.3(24.5-51.6)$ & $41.9(24.5-51.6)$ & $43.0(31-50.8)$ & 0.336 & $42.2(27.8-51.6)$ & $42.4(24.5-50.8)$ & 0.850 \\
\hline Hemoglobin ( $g / L)$ & $143.9(63-181)$ & $143.9(63-181)$ & $144.0(108-75)$ & 0.996 & $143.2(63-181)$ & 144.9 (104-176) & 0.747 \\
\hline Platelets (10^9/L) & $150.8(26-315)$ & $151.7(26-315)$ & $148.4(57-246)$ & 0.850 & $131.9(26-315)$ & $176.6(49-308)$ & 0.004 \\
\hline $\operatorname{AFP}(\mathrm{ng} / \mathrm{mL})$ & $13.9(2.0-147.2)$ & II.7 (2.0-80.7) & $18.5(2.1-147.2)$ & 0.342 & $17.5(2.2-147.2)$ & $8.5(2.0-18.6)$ & 0.112 \\
\hline HCV-RNA (lg IU/mL) & $6.20(3.21-7.91)$ & $6.17(3.21-7.91)$ & $6.24(3.78-7.79)$ & 0.766 & $6.13(4.06-7.79)$ & $6.29(3.21-7.91)$ & 0.461 \\
\hline APRI & $2.36(0.18-17.17)$ & $2.37(0.18-17.17)$ & $2.32(0.46-11.05)$ & 0.951 & $3.01(0.20-17.17)$ & $1.40(0.18-5.14)$ & 0.007 \\
\hline FIB-4 & $3.67(0.25-21.05)$ & $3.68(0.25-21.05)$ & $3.64(0.53-12.38)$ & 0.967 & $4.38(0.39-21.05)$ & $2.61(0.25-12.11)$ & 0.040 \\
\hline Cirrhosis, n (\%) & $33(32.7 \%)$ & $23(34.8 \%)$ & $10(28.6 \%)$ & 0.657 & $24(40.7 \%)$ & $9(21.4 \%)$ & 0.042 \\
\hline Treatment experienced ${ }^{\mathrm{a}}, \mathrm{n}(\%)$ & $8(7.9 \%)$ & $7(10.6 \%)$ & I (2.9\%) & 0.256 & $6(10.2 \%)$ & $2(4.8 \%)$ & 0.464 \\
\hline HBV coinfection, n (\%) & $6(5.9 \%)$ & $5(7.6 \%)$ & I (2.9\%) & 0.662 & $5(8.5 \%)$ & I (2.4\%) & 0.396 \\
\hline \multicolumn{8}{|l|}{ Treatment regimens } \\
\hline SOF $\pm R B V, n(\%)$ & $28(27.7 \%)$ & $17(25.8 \%)$ & II (31.4\%) & 0.377 & $14(23.7 \%)$ & $14(33.3 \%)$ & 0.414 \\
\hline SOF+DCV $\pm R B V, n(\%)$ & $19(18.8 \%)$ & $15(22.7 \%)$ & 4 (II.4\%) & & $13(22.0 \%)$ & $6(14.3 \%)$ & \\
\hline SOF/VEL $\pm R B V, n(\%)$ & $54(53.5 \%)$ & $34(51.5 \%)$ & $20(57.1 \%)$ & & $32(54.2 \%)$ & $22(52.4 \%)$ & \\
\hline \multicolumn{8}{|l|}{ Genotype } \\
\hline Genotype 3, n (\%) & $59(58.4 \%)$ & $45(68.2 \%)$ & $14(40.0 \%)$ & 0.006 & & & \\
\hline Genotype 6, n (\%) & $42(41.6 \%)$ & 21 (31.8\%) & 21 (60.0\%) & & & & \\
\hline \multicolumn{8}{|l|}{ RBV coadministration } \\
\hline With & & & & & 45 (76.3\%) & $21(50.0 \%)$ & 0.010 \\
\hline Without & & & & & $14(23.7 \%)$ & $2 \mathrm{I}(50.0 \%)$ & \\
\hline
\end{tabular}

Note: ${ }^{\text {aTeated }}$ with DAAs or interferon and ribavirin regimens.

Abbreviations: AFP, alpha fetoprotein; ALT, alanine aminotransferase; APRI, aspartate aminotransferase to platelet ratio index; AST, aspartate aminotransferase; DCV, daclatasvir; FIB-4, fibrosis-4; HBV, hepatitis B virus; HCV, hepatitis C virus; RBV, ribavirin; RNA, ribonucleic acid; SOF, sofosbuvir; VEL, velpatasvir.

of ALT, AST, total bilirubin, total bile acid, albumin, hemoglobin, AFP, serum HCV-RNA, proportion of HBV coinfection or treatment experience, except for higher scores of APRI and FIB-4, lower levels of platelets, higher proportions of cirrhosis, and RBV coadministration in patients with genotype 3 infection.

\section{High Virologic Response in Patients Infected with Genotypes 3 and 6}

The data in Figure 1 show patient disposition and the SVR12 outcomes of various treatment regimens and genotypes. Overall, the SVR12 rate was $95.0 \%$ (96/101) among all patients, $93.2 \%$ (55/59) among patients infected with genotype 3 , and $97.6 \%$ (41/ 42) among patients infected with genotype 6. Among patients infected with genotype 3, the SVR12 rates were achieved in $77.8 \%, 100 \%$, and $96 \%$ among patients treated with SOF+RBV, $\mathrm{SOF}+\mathrm{DCV}+\mathrm{RBV}$, and $\mathrm{SOF} / \mathrm{VEL}+\mathrm{RBV}$ regimens, respectively, compared to $100 \%, 100 \%$, and $85.7 \%$ without RBV regimens, respectively. Similar results were found in patients infected with genotype 6, where the SVR12 rates were achieved in $100 \%$, $100 \%$ and $100 \%$ among patients treated with SOF+RBV, SOF $+\mathrm{DCV}+\mathrm{RBV}$, and SOF/VEL+RBV, respectively, compared to 


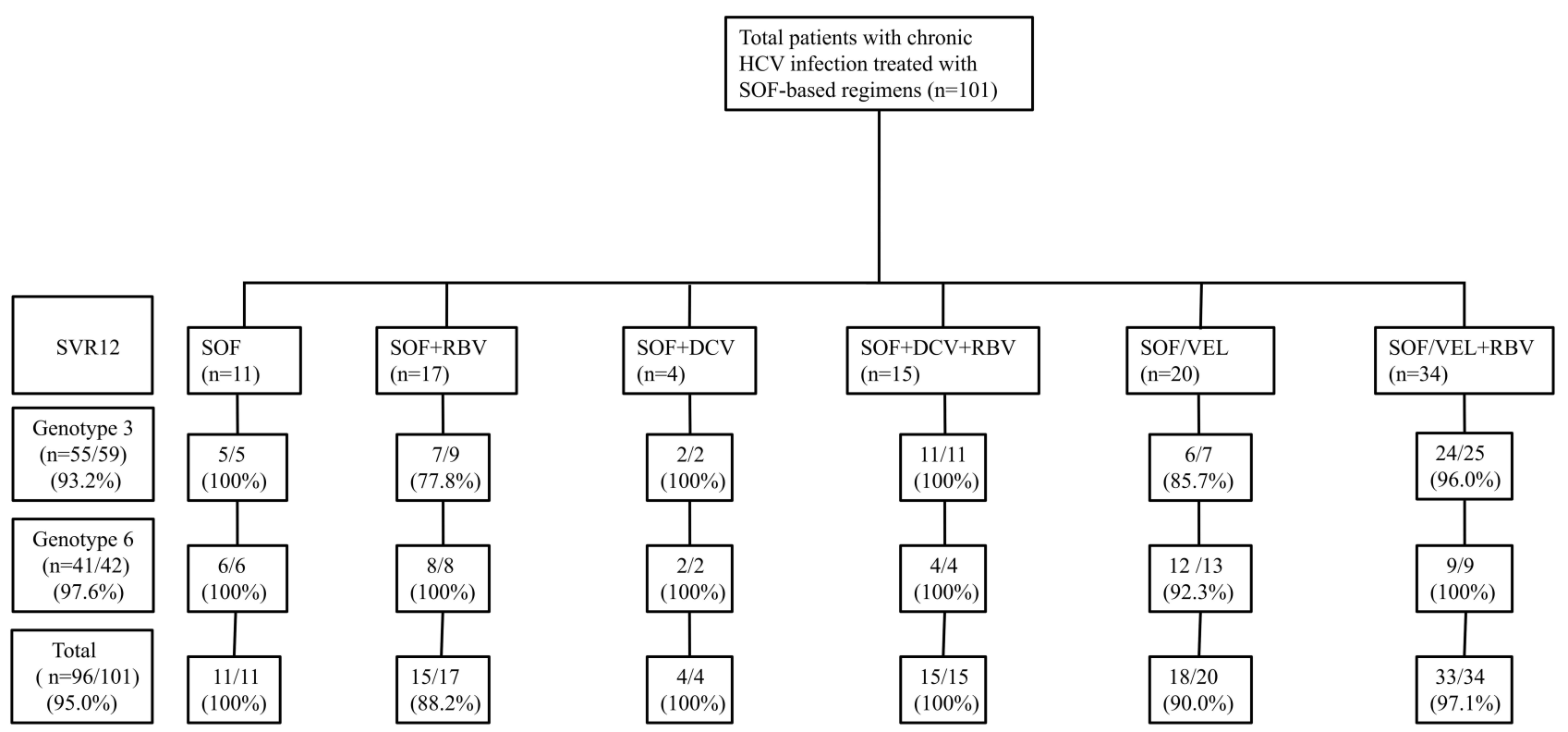

Figure I Patient disposition and SVRI2 rates in various treatment regimens and genotypes. Study flow design. Distribution of patients into the overall cohort and sustained virological response at posttreatment week 12 (SVRI2) rates in diverse genotypes and treatment regimens.

$100 \%, 100 \%$, and $92.3 \%$ without RBV regimens, respectively. The SVR12 rates of the SOF+RBV and SOF/VEL regimens were numerically lower than those of the other regimens; however, no significant difference was found (Supplementary Figure 1). Of note, the proportion of cirrhotic patients for each treatment regimen was similar $(\mathrm{p}=0.368)$.

We further analyzed the impact of factors such as sex, age, cirrhotic status, viral load, treatment history, HBV coinfection, and level of baseline ALT on SVR12 in patients with genotype 3 and 6 infections. The data in Figure 2 show that the above mentioned factors had no impact on SVR12 in either genotype 3 or 6.

\section{High SVRI2 Rate in Subtype 3b}

Patients infected with the subtype 3 b achieved an SVR12 rate of $92.1 \%(35 / 38)$, which accounted for similar and high

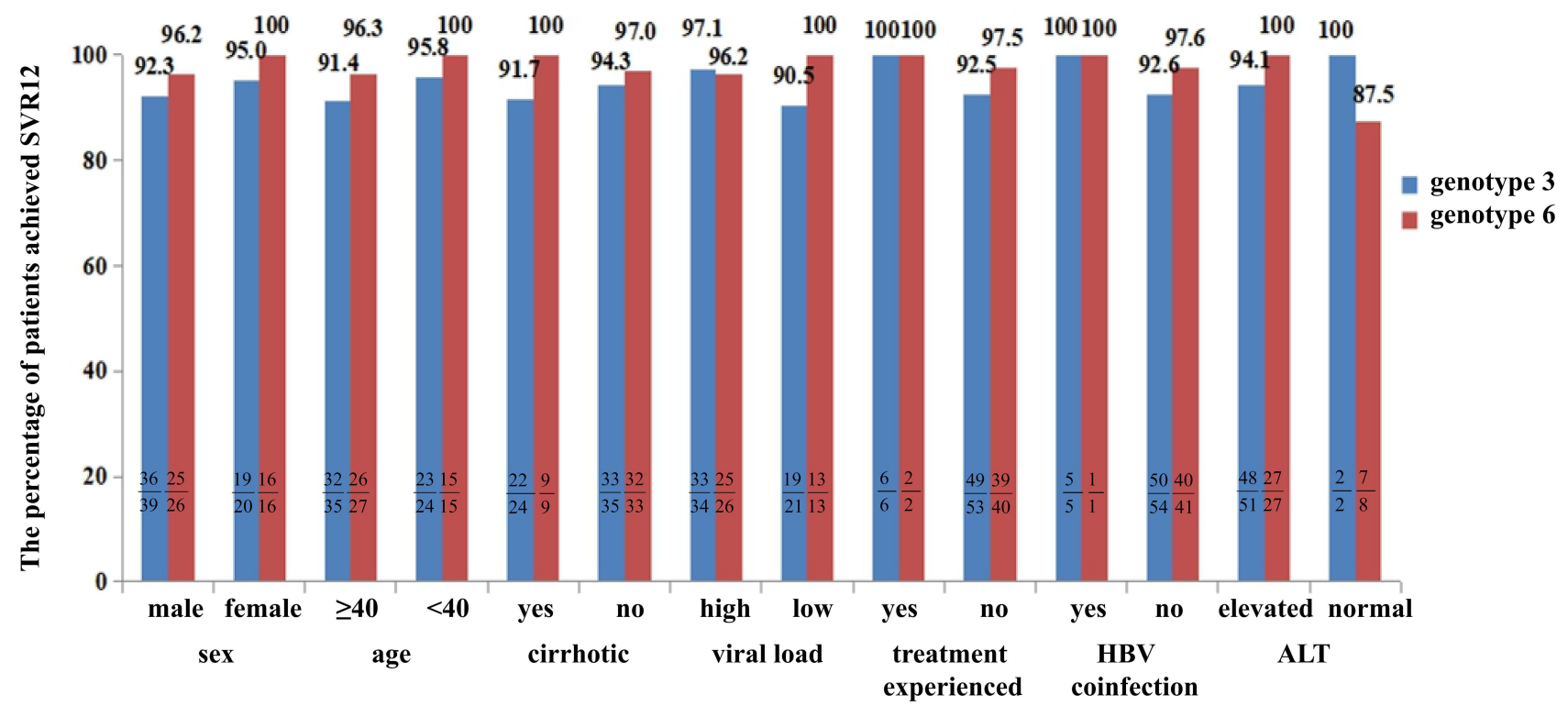

Figure 2 SVR 12 rates in patients infected with genotypes 3 and 6 with different characteristics. We analyzed the impact of factors such as sex, age, cirrhotic status, viral load (high level of viral load: serum HCV-RNA> $800,000 \mathrm{IU} / \mathrm{mL}$, low level of viral load: HCV-RNA $\leq 800,000 \mathrm{IU} / \mathrm{mL}$ ), treatment history, HBV coinfection and level of baseline ALT on SVRI 2 in patients with genotypes 3 and 6 infections. For genotype 3, no significant difference was found between the above factors: $p>0.05$. Similar results were found in patients infected with genotype 6 . 
SVR12 rates irrespective of sex, age, cirrhosis status, treatment history, viral load, HBV coinfection, and level of baseline ALT (Figure 3). Moreover, the data in Supplementary Figure 2 shows no significant difference in the SVR12 rates between the $3 \mathrm{~b}$ and non $3 \mathrm{~b}$ genotypes. (92.1\% versus $95.2 \%$, respectively, $\mathrm{p}=1.000)$. Among the cirrhotic patients infected with the subtype $3 b$, the SVR12 rate was $93.3 \%$ (14/15), and $80.0 \%$ of these patients were treated with RBV to SOF-based regimens. The detailed clinical process of those patients is described in Figure 4, except that one patient who was treated with $\mathrm{SOF} / \mathrm{VEL}+\mathrm{RBV}$ for 12 weeks was not presented because the time of undetectable HCV-RNA was unclear.

\section{Patients Failed to Achieve SVRI2}

In total, five patients with good compliance failed to achieve SVR12. All of these patients were treated naively, including 2 noncirrhotic patients with genotype $3 \mathrm{~b}$ infection treated with $\mathrm{SOF}+\mathrm{RBV}$ and $\mathrm{SOF} / \mathrm{VEL}+\mathrm{RBV}, 2$ cirrhotic patients with genotype $3 \mathrm{~b}$ and $3 \mathrm{k}$ infection treated with $\mathrm{SOF}+\mathrm{RBV}$ and SOF/VEL, and 1 noncirrhotic patient with 6a infection treated with SOF/VEL. The detailed characteristics of those patients are presented in Supplementary Table 1.

\section{Numerically Higher SVR I 2 Rate in RBV Coadministration Regimens}

The baseline characteristics of patients treated with RBV are presented in Table 1. Patients treated with RBV coadministration to SOF-based regimens achieved an SVR12 rate of $95.5 \%(63 / 66)$, which was similar to the rate of 94.3\% (33/35) found in the RBV-free regimen ( $\mathrm{P}=1.000)$. We compared the SVR12 rates in patients treated with or without RBV in diverse characteristics, such as genotype, viral load, cirrhosis status, HBV coinfection, and treatment history. Finally, RBV coadministration was found to mildly increase the SVR12 rate; however, no significant difference was found. (Figure 5)

Of note, the SVR12 rate was $93.9 \%$ in cirrhotic patients (31/33), 95.7\% in those with RBV coadministration regimens, and $90.0 \%$ in those without RBV $(\mathrm{p}=0.521)$. Among cirrhotic patients infected with genotype 3, the overall SVR12 rate was $91.7 \%$ (22/24); specifically, the rate was $95.0 \%$ in those with ribavirin coadministration regimens, which was numerically higher than the rate of $75.0 \%$ found in those without ribavirin, but no significant difference was found $(\mathrm{p}=0.312)$.

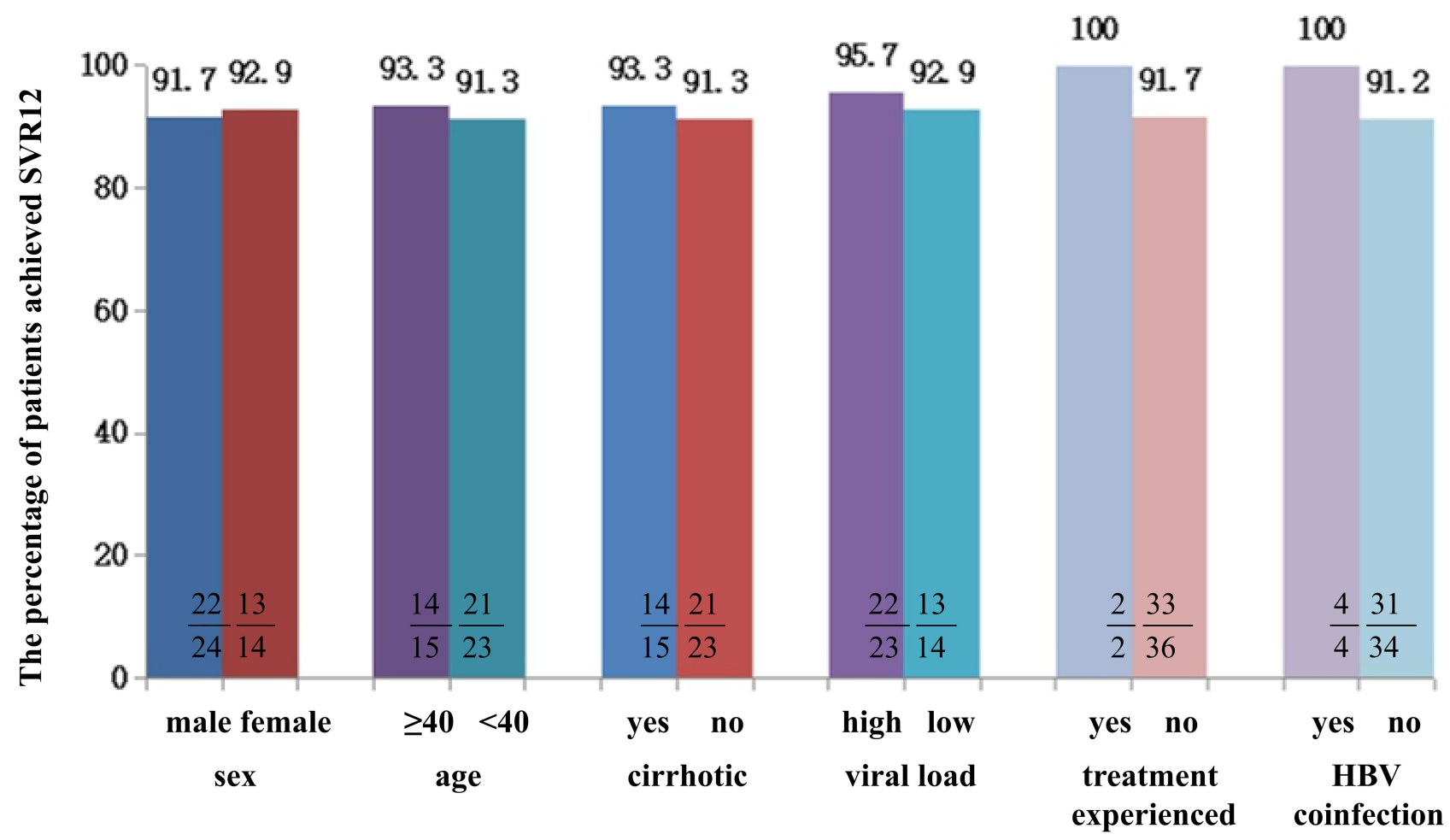

Figure 3 SVRI 2 rates in patients infected with genotype $3 \mathrm{~b}$ with different characteristics. We analyzed the impact of factors such as sex, age, cirrhotic status, viral load, treatment history, HBV coinfection and level of baseline ALT on SVRI 2 in patients with genotype $3 \mathrm{~b}$ infection. No significant difference was found between the above factors: $p>0.05$. 


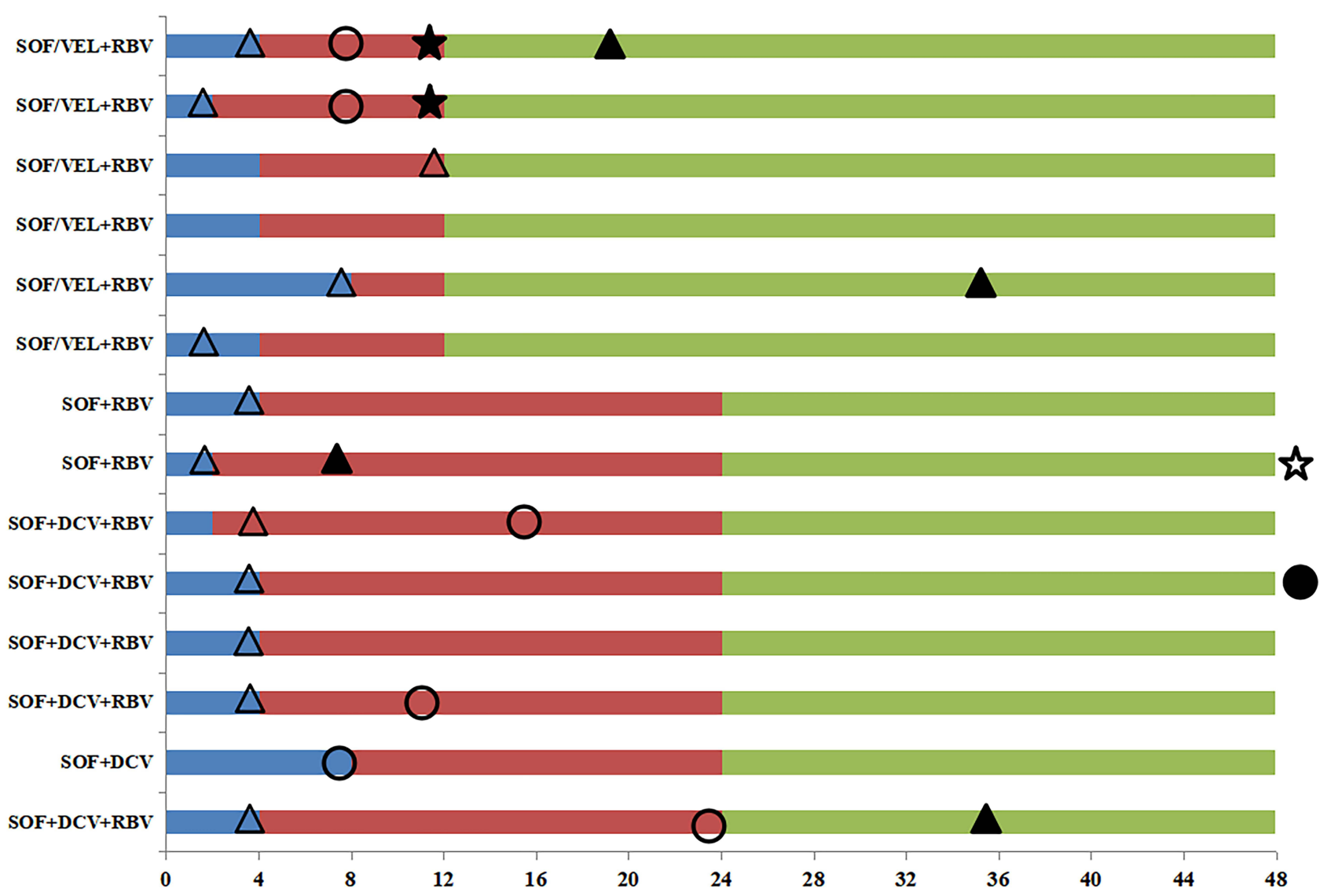

Figure 4 The detailed clinical process in cirrhotic patients with genotype $3 \mathrm{~b}$ infection.

Notes: -Serum HCV-RNA detectable -Time at serum HCV-RNA undetectable to end of treatment. -Follow up after end of treatment. $\triangle$-Time at occurrence of ALT normalization. $O$-Time at occurrence of AFP normalization. -Time at occurrence of elevated ALT which was normal before. - -Patient whose AFP was normal before but elevated during follow-up. $\$$-Time at detectable serum HCV-RNA which was undetectable before. 7 -Patient who achieved SVR I 2 but detectable serum HCVRNA during follow-up. Each bar represents a patient. The left side of the figure shows the treatment regimens, the horizontal axis represents the time (week), and week 0 indicates the time at baseline. The number of cirrhotic patients infected with genotype $3 \mathrm{~b}$ was 15 , and one patient treated with SOF/VEL+RBV for 12 weeks was not presented because the time of undetectable HCV-RNA was unclear.

\section{Antiviral Treatment Contributes to Fibrosis Improvement}

After a median follow-up of 15.2 months, the mean APRI and FIB-4 scores decreased significantly. The data in Figure 6 show that the APRI was significantly decreased compared to that at baseline ( 2.25 versus $0.58, \mathrm{p}<0.0001)$, and a similar result was found in FIB-4 (3.55 versus 2.13, $\mathrm{p}<0.0001)$. We further compared the APRI and FIB-4 scores between pretreatment and posttreatment among patients with different characteristics (Supplementary Table 2). The outcomes indicated that patients with HBV coinfection, treatment experience, and normal ALT levels at baseline and those who failed to achieve the SVR12 gained no significant decrease in APRI or FIB-4 scores. The multivariate logistic regression (Table 2) indicated that a high viral load (HCV-RNA>800,000 IU/mL) level at baseline was identified as a negative predictor of fibrosis improvement $(\mathrm{p}=0.023, \mathrm{OR}=5.695(1.275-25.433)$ ).

\section{SOF-Based Regimens Were Well-Tolerated}

SOF-based treatment was well-tolerated by patients. The data in Table 3 show that adverse events (AEs) occurred in $12.9 \%$ of patients, that no severe AEs or deaths occurred, and that there was no discontinuation due to AEs. Furthermore, there was no significant difference in the 6 kinds of treatment regimens $(\mathrm{P}=0.273)$. Additionally, there was no significant difference in the incidence of AEs between RBV coadministration and RBV-free treatment regimens $(12.1 \%$ versus $14.3 \%, \mathrm{P}=0.757)$.

\section{Discussion}

In the present study, we assessed whether SOF-based regimens achieved satisfactory virological response and were well tolerated in Chinese patients infected with genotype 3 and 6 in real-world practice. Although a higher proportion of 


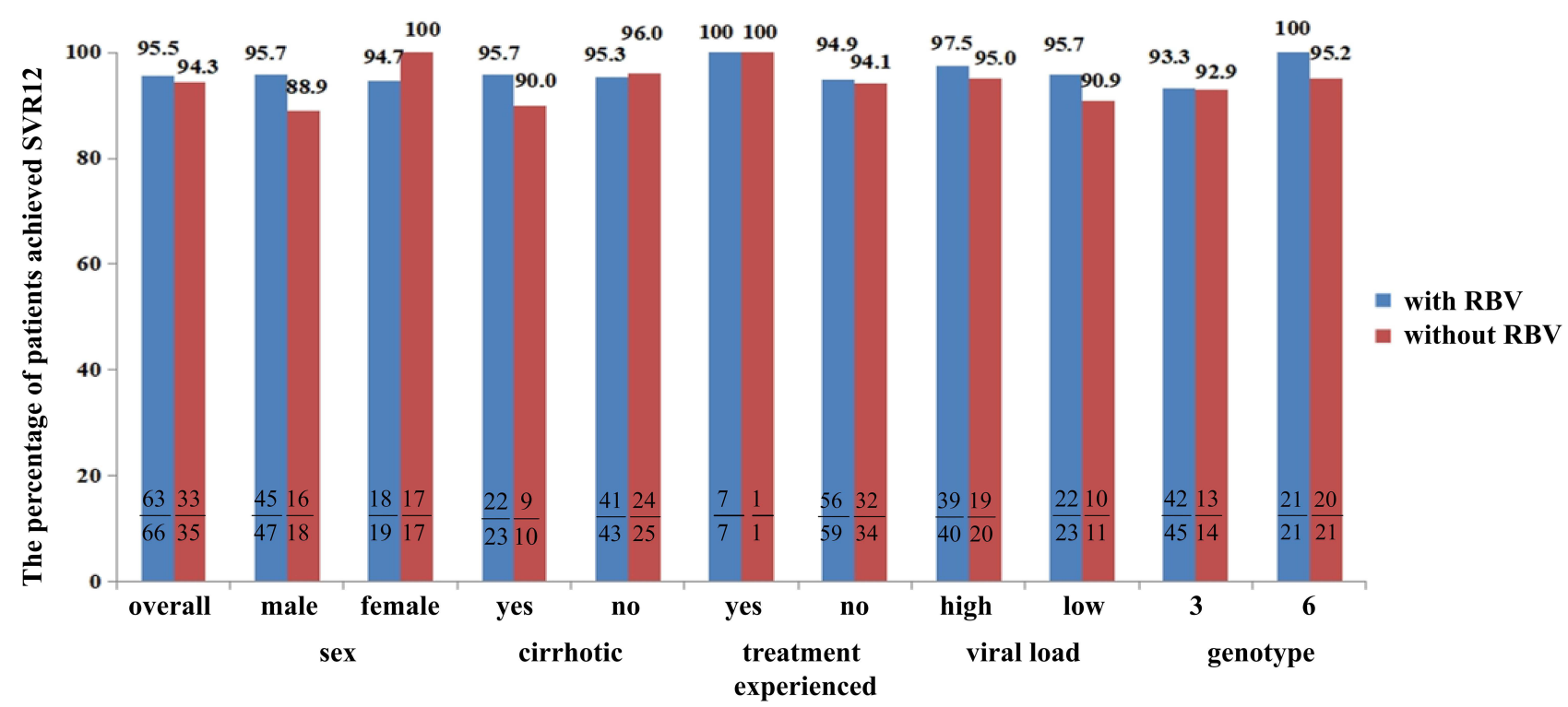

Figure 5 SVRI2 rates in patients treated with or without RBV with different characteristics. We compared the SVRI2 rates in cirrhotic, noncirrhotic, treatmentexperienced (treated with DAAs or interferon and RBV regimens), treatment-naive, high viral load (level of serum HCV-RNA $>800,000$ IU/mL), low viral load (HCV-RNA $\leq$ $800,000 \mathrm{lU} / \mathrm{mL}$ ), and two genotypes. In patients with the above characteristics, no significant difference was found between the groups with RBV and without RBV: $>>0.05$.
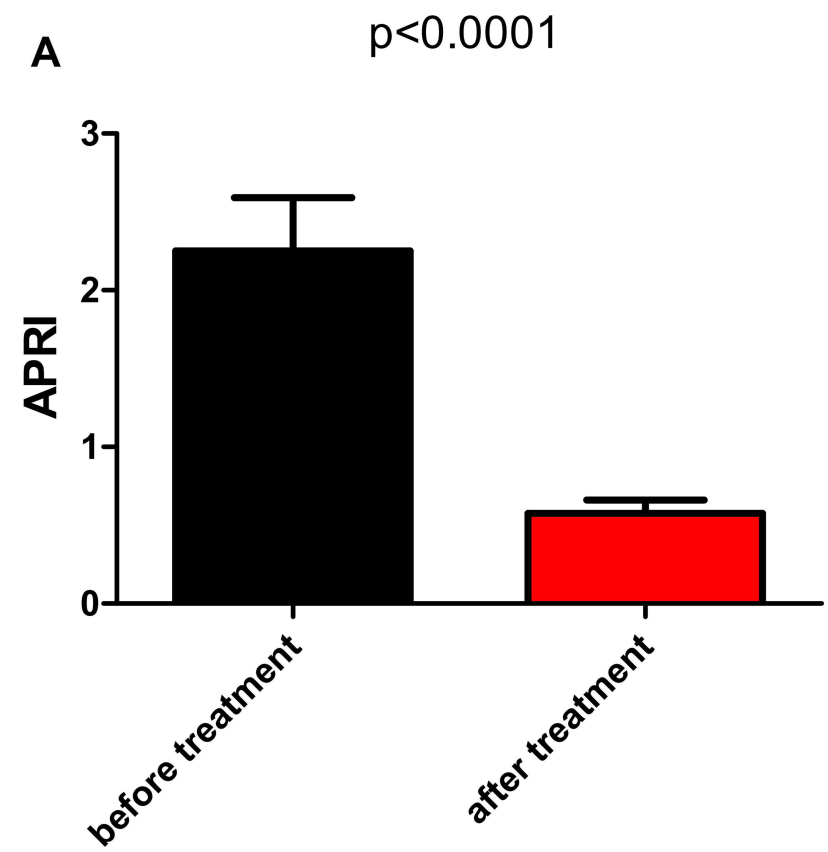

B
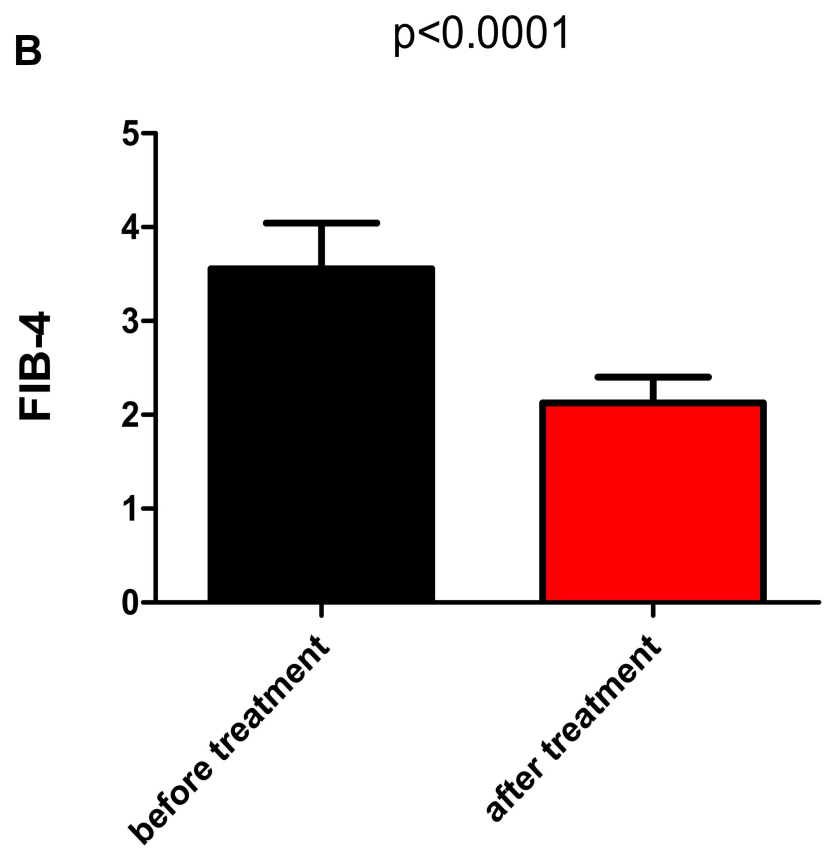

Figure 6 The improvement in fibrosis evaluated by APRI and FIB4. Sub-figure 6A compared the APRI (AST/PLT*I00) and Sub-figure 6B compared the FIB-4 ((Age $\times$ AST)/ $(\mathrm{PLT} \times($ square root of $\mathrm{ALT}))$ between pretreatment and posttreatment. The difference in APRI between pretreatment and posttreatment: $\mathrm{P}<0.000 \mathrm{I}$; a similar result was found in FIB-4.

cirrhosis and higher levels of APRI and FIB-4 at baseline were found in patients infected with genotype 3 compared to those infected with genotype 6 , similar and high SVR12 rates were estimated in the two genotypes. Additionally, the SVR12 rates were similar and high in diverse SOF-based regimens. The addition of $\mathrm{RBV}$ treatments yielded numerically higher SVR12 rates compared to RBV-free regimens in cirrhotic patients infected with genotype 3 or $3 b$.

SOF (an NS5B nucleotide polymerase inhibitor), DCV (an NS5A replication complex inhibitor, which is not FDAapproved but has been studied widely), and SOF/VEL (a new pangenotypic drug, which is the combination of NS5A 
Table 2 Univariate and Multivariate Logistic Analysis of Factors Associated with Fibrosis Improvement

\begin{tabular}{|c|c|c|c|c|}
\hline Factors & & Univariate $P$ value & Multivariate $P$ value & Odds Ratio (95\% Cl) \\
\hline Sex & $\begin{array}{l}\text { Male } \\
\text { Female }\end{array}$ & 0.183 & & \\
\hline Age & $\begin{array}{l}\leq 40 y \\
>40 y\end{array}$ & 0.054 & 0.386 & $0.468(0.084-2.605)$ \\
\hline Cirrhotic & $\begin{array}{l}\text { Yes } \\
\text { No }\end{array}$ & 0.155 & & \\
\hline Viral load ${ }^{\mathrm{a}}$ & $\begin{array}{l}\text { High } \\
\text { Low }\end{array}$ & 0.036 & 0.023 & $5.695(1.275-25.433)$ \\
\hline Treatment experienced $^{\mathrm{b}}$ & $\begin{array}{l}\text { Yes } \\
\text { No }\end{array}$ & 0.596 & & \\
\hline HBV coinfection & $\begin{array}{l}\text { Yes } \\
\text { No }\end{array}$ & 0.717 & & \\
\hline ALT level & $\begin{array}{l}\text { Elevated } \\
\text { Normal }\end{array}$ & 0.736 & & \\
\hline Genotype & $\begin{array}{l}3 \\
6\end{array}$ & 0.558 & & \\
\hline Treatment regimens & $\begin{array}{l}\text { SOF } \pm R B V \\
\text { SOF+DCV } \pm R B V \\
\text { SOF/VEL } \pm R B V\end{array}$ & 0.984 & & \\
\hline RBV addition & $\begin{array}{l}\text { With } \\
\text { Without }\end{array}$ & 0.282 & & \\
\hline APRI & & 0.036 & 0.510 & $0.669(0.142-3.141)$ \\
\hline FIB-4 & & 0.029 & 0.330 & $0.686(0.224-2.101)$ \\
\hline SVRI2 & $\begin{array}{l}\text { Yes } \\
\text { No }\end{array}$ & 0.999 & & \\
\hline
\end{tabular}

Notes: ${ }^{a}$ High level of viral load $(\mathrm{HCV}-\mathrm{RNA}>800,000 \mathrm{IU} / \mathrm{mL})$ and low level of viral load $(\mathrm{HCV}-\mathrm{RNA} \leq 800,000 \mathrm{IU} / \mathrm{mL}) .{ }^{\mathrm{b}}$ Treated with DAAs or interferon and ribavirin regimens.

Abbreviations: ALT, alanine aminotransaminase; APRI, aspartate aminotransferase to platelet ratio index; DCV, daclatasvir; FIB-4, fibrosis-4; HBV, hepatitis B virus; SOF, sofosbuvir; SVRI2, sustained virological response at posttreatment week I2; RBV, ribavirin; VEL, velpatasvir.

and NS5B nucleotide polymerase inhibitor) are highly effective in HCV infection and are approved for treatment among patients with genotype 3 and 6 infections, according to the guidelines and clinical trials. ${ }^{15,26}$ However, it has been reported that SVR12 rates in real-world practice are not the same as those in clinical trials. ${ }^{27}$ A Phase III clinical trial from Asia showed an unsatisfactory SVR12 rate of SOF/VEL treatment in patients infected with genotype $3 \mathrm{~b}$, which was significantly inferior to other genotypes, especially in cirrhotic patients, whose SVR12 rate was only $50 \% .{ }^{28}$ In China, to our knowledge, only a few studies based on real-world practice have explored the efficacy of SOF-based regimens, particularly SOF/VEL, in patients with genotype 3 and 6 infections.
One study conducted by Tang H provided data on the efficacy of the SOF-based regimens in Chinese patients infected with genotype $3(n=60)$. The SVR24 rate was over $90 \%$ in the SOF $+\mathrm{DCV}+\mathrm{RBV}$ and SOF/VEL regimens, but it was not over $90 \%$ in the SOF+DCV regimens. Furthermore, the subgenotype information was not provided, and the effect of ribavirin on SVR12 was not analyzed. ${ }^{29}$ Another real-world study from China showed that genotype 3a-infected patients yield high and similar SVR12 rates in SOF/VEL and SOF $+\mathrm{DCV} \pm \mathrm{RBV}$ regimens. ${ }^{30}$ However, the number of patients enrolled was small, and the SVR12 rate in the subtype 3b with cirrhosis was not shown. In the present study, patients with genotype 3 infection achieved SVR12 rates over $90 \%$, with some even 
Table 3 The Adverse Events of Patients Who Received SOF-Based Regimens

\begin{tabular}{|c|c|c|c|c|c|c|c|c|}
\hline & $\begin{array}{l}\text { Total } \\
(n=101)\end{array}$ & $\begin{array}{l}\text { SOF } \\
(n=I I)\end{array}$ & $\begin{array}{l}\text { SOF+RBV } \\
(n=\mid 7)\end{array}$ & $\begin{array}{l}\text { SOF+DCV } \\
(n=4)\end{array}$ & $\begin{array}{l}\text { SOF+DCV } \\
+R B V(n=\mid 5)\end{array}$ & $\begin{array}{l}\text { SOF/VEL } \\
(n=20)\end{array}$ & $\begin{array}{l}\text { SOF/VEL } \\
+\operatorname{RBV}(n=34)\end{array}$ & $P$ value \\
\hline AEs, n (\%) & $13(12.9)$ & $2(I 8.2)$ & $2(11.8)$ & $2(50.0)$ & $2(13.3)$ & I (5.0) & $4(11.8)$ & 0.273 \\
\hline $\begin{array}{l}\text { Mild to moderate } \\
\text { anemia }\end{array}$ & $8(7.9)$ & $2(18.2)$ & I (5.9) & $0(0)$ & I (6.7) & I (5.0) & $3(8.8)$ & 0.754 \\
\hline Severe anemia & $\mathrm{I}(\mathrm{I} .0)$ & $0(0)$ & $0(0)$ & $0(0)$ & $0(0)$ & $0(0)$ & I (2.9) & \\
\hline Abdominal distention & $2(2.0)$ & $0(0)$ & I (5.9) & $0(0)$ & I (6.7) & $0(0)$ & $0(0)$ & 1.000 \\
\hline Fatigue, n (\%) & I (I.0) & $0(0)$ & $0(0)$ & I (25.0) & $0(0)$ & $0(0)$ & $0(0)$ & \\
\hline Headache, n (\%) & I (I.0) & $0(0)$ & $0(0)$ & I (25.0) & $0(0)$ & $0(0)$ & $0(0)$ & \\
\hline Diarrhea, n (\%) & $0(0)$ & $0(0)$ & $0(0)$ & $0(0)$ & $0(0)$ & $0(0)$ & $0(0)$ & \\
\hline Serious AEs, n (\%) & $0(0)$ & $0(0)$ & $0(0)$ & $0(0)$ & $0(0)$ & $0(0)$ & $0(0)$ & \\
\hline $\begin{array}{l}\text { Discontinuation due } \\
\text { to AEs, } n(\%)\end{array}$ & $0(0)$ & $0(0)$ & $0(0)$ & $0(0)$ & $0(0)$ & $0(0)$ & $0(0)$ & \\
\hline Death, n (\%) & $0(0)$ & $0(0)$ & $0(0)$ & $0(0)$ & $0(0)$ & $0(0)$ & $0(0)$ & \\
\hline
\end{tabular}

Abbreviations: AEs: adverse events; DCV, daclatasvir; RBV, ribavirin; SOF, sofosbuvir; VEL, velpatasvir.

higher than $100 \%$, in the $\mathrm{SOF}$ and $\mathrm{SOF}+\mathrm{DCV} \pm \mathrm{RBV}$ regimens. Among cirrhotic patients infected with genotype $3 \mathrm{~b}$ $(\mathrm{n}=15)$, the SVR12 rate was $93.3 \%$.

RBV has been shown to improve the efficacy of pegylated interferon, which has been recommended in patients with decompensated cirrhosis at the time of DAA therapy, according to guidelines. ${ }^{26,31}$ The role of RBV in antiviral efficacy in DAA therapy is still controversial. Data from a systematic review has shown that the addition of RBV to SOF/VEL and SOF +DCV significantly increases the efficacy and side effects. ${ }^{10}$ Several studies have reported that RBV coadministration has no impact on antiviral efficacy. ${ }^{18,19}$ In the present study, RBV was more frequently used in patients with genotype 3 infection than those with genotype 6 infection, but they obtained similar and high SVR12 rates and side effects. Additionally, RBV coadministration to three SOF-based regimens achieved a numerically higher SVR12 rate irrespective of genotype, viral load, and treatment experience. Additionally, in cirrhotic patients infected with genotype 3 or $3 \mathrm{~b}$ and compared to RBV-free regimens, RBV coadministration numerically increased the SVR12 rate without significantly increasing the side effects. Moreover, there is evidence for an increased occurrence of HCC in patients treated with RBV-free SOF-based regimens. ${ }^{32}$ Accordingly, this may provide evidence for the treatment of genotypes 3 and 6 in China; that is, RBV coadministration to SOF-based regimens should be applied for more satisfactory SVR12 rates and lower incidence rates of HCC. Likewise, its application helps contribute to achieving the ambitious goal of eliminating HCV by 2030.

It is well known that antiviral treatment contributes to fibrosis improvement. Data from a long-term follow-up study showed that high ALT at baseline predicts fibrosis progression. $^{33}$ A real-world study from China first reported that liver fibrosis evaluated by APRI and FIB-4 was significantly improved in patients with genotype 3 infection. ${ }^{29}$ In the present study, we also found that fibrosis improvement evaluated by APRI and FIB-4 occurred in most patients after a median follow-up of 15.2 months, including cirrhotic patients or those who achieved SVR12; however, such improvement was not found in patients with HBV co-infection, those with treatment experience, normal those with a level of ALT at baseline or those who failed to achieve SVR12, which are opposed to those mentioned in the above study. Moreover, we found that a high viral load at baseline was a negative predictor of fibrosis improvement. In our opinion, it is an issue of great concern to monitor cirrhotic status for patients with the following characteristics: treatment experience, HBV coinfection, normal level of ALT, or high viral load at baseline. 
More research should be conducted to identify the predictors of fibrosis progression.

There were some limitations in the present retrospective study. The number of patients included was insufficient, which means that most of the groups compared had fewer than 10 patients each, and it was therefore difficult to find significant differences. Only $87 \%$ of the patients had a serum viral load detected with Roche Cobas Ampliprep/Cobas TaqMan, which has a lowest limit of quantification (LLOQ) value of $15 \mathrm{IU} / \mathrm{mL}$ at 12 weeks posttreatment, or with Kehua detection, which has an LLOQ value of $1000 \mathrm{IU} / \mathrm{mL}$ at 24 weeks posttreatment. For patients who failed to achieve SVR12, we did not take further action to confirm the reasons, such as alcohol intake and drug resistance. We did not use liver biopsy to assess the degree of liver fibrosis improvement, which may have affected the results.

\section{Conclusion}

Real-world data show that sofosbuvir-based regimens are highly effective and safe for patients with HCV genotype 3 and 6 infections.

\section{Abbreviations}

HCV, Hepatitis C virus; SOF, Sofosbuvir; SVR, Sustained virological response; DAA, Direct-acting antivirals; RBV, Ribavirin; VEL, Velpatasvir; DCV, Daclatasvir; HBV, Hepatitis B virus; AST, Aspartate aminotransferase; ALT, Alanine aminotransaminase; APRI, Aspartate aminotransferase to platelet ratio index; FIB-4, Fibrosis-4; AE, Adverse event; LLOQ, Lower limit of quantification.

\section{Data Sharing Statement}

The datasets used and analyzed during the current study are available from the corresponding author upon reasonable request.

\section{Ethical Approval}

This study was approved by the Ethical Committee of the Second Affiliated Hospital of Chongqing Medical University. This study was conducted in accordance with the Declaration of Helsinki. The ethical approval number is 2019 (30).

\section{Informed Consent}

We obtained informed consent forms signed by all participants.

\section{Consent for Publication}

We obtained approval for publication from the participants.

\section{Acknowledgments}

The authors are grateful to Professor Peng $\mathrm{Hu}$ for the assistance in designing the study and to Li Wei and Xiaoqing Liu for assisting with the procedures and discussing the results.

\section{Author Contributions}

PH conceived the study, LW and XQL retrieved and analyzed the data, and all the authors contributed to the writing of the manuscript. All authors made substantial contributions to conception and design, acquisition of data, or analysis and interpretation of data; took part in drafting the article or revising it critically for important intellectual content; agreed to submit to the current journal; gave final approval of the version to be published; and agree to be accountable for all aspects of the work.

\section{Funding}

This work was prepared independently without financial support.

\section{Disclosure}

The authors report no conflicts of interest in this work.

\section{References}

1. Messina JP, Humphreys I, Flaxman A, et al. Global distribution and prevalence of hepatitis C virus genotypes. Hepatology. 2015;61 (1):77-87. doi:10.1002/hep.27259

2. Ju W, Yang S, Feng S, et al. Hepatitis C virus genotype and subtype distribution in Chinese chronic hepatitis $\mathrm{C}$ patients: nationwide spread of HCV genotypes 3 and 6. Virol J. 2015;12(1):109. doi:10.1186/ s12985-015-0341-1

3. Rao H, Wei L, Lopez-Talavera JC, et al. Distribution and clinical correlates of viral and host genotypes in Chinese patients with chronic hepatitis C virus infection. J Gastroenterol Hepatol. 2014;29 (3):545-553. doi:10.1111/jgh.12398

4. Zhang Y, Chen LM, He M. Hepatitis C Virus in mainland China with an emphasis on genotype and subtype distribution. Virol J. 2017;14 (1):41. doi:10.1186/s12985-017-0710-z

5. Lee MH, Yang HI, Lu SN, et al. Chronic hepatitis C virus infection increases mortality from hepatic and extrahepatic diseases: a community-based long-term prospective study. $J$ Infect Dis. 2012;206(4):469-477. doi:10.1093/infdis/jis385

6. Backus LI, Boothroyd DB, Phillips BR, et al. A sustained virologic response reduces risk of all-cause mortality in patients with hepatitis C. Clin Gastroenterol Hepatol. 2011;9(6):509-516 e501. doi:10.1016/ j.cgh.2011.03.004

7. Nkontchou G, Ziol M, Aout M, et al. HCV genotype 3 is associated with a higher hepatocellular carcinoma incidence in patients with ongoing viral C cirrhosis. J Viral Hepat. 2011;18(10):e516-522. doi:10.1111/j.1365-2893.2011.01441.x 
8. Kanwal F, Kramer JR, Ilyas J, et al. HCV genotype 3 is associated with an increased risk of cirrhosis and hepatocellular cancer in a national sample of U.S. Veterans with HCV. Hepatology. 2014;60 (1):98-105. doi:10.1002/hep.27095

9. Lee MH, Hsiao TI, Subramaniam SR, et al. HCV genotype 6 increased the risk for hepatocellular carcinoma among asian patients with liver cirrhosis. Am J Gastroenterol. 2017;112(7):1111-1119. doi:10.1038/ajg.2017.123

10. Berden FA, Aaldering BR, Groenewoud H, et al. Identification of the best direct-acting antiviral regimen for patients with hepatitis $\mathrm{c}$ virus genotype 3 infection: a systematic Review and network meta-analysis. Clin Gastroenterol Hepatol. 2017;15(3):349-359. doi:10.1016/j.cgh.2016.10.034

11. Hu C, Yuan G, Liu J, et al. Sofosbuvir-based therapies for patients with hepatitis $\mathrm{C}$ virus infection: real-world experience in China. Can J Gastroenterol Hepatol. 2018;2018:3908767. doi:10.1155/2018/ 3908767

12. Gayam V, Tiongson B, Khalid M, et al. Sofosbuvir based regimens in the treatment of chronic hepatitis $\mathrm{C}$ genotype 1 infection in African-American patients: a community-based retrospective cohort study. Eur J Gastroenterol Hepatol. 2018;30(10):1200-1207. doi:10.1097/MEG.0000000000001233

13. Wu DB, Jiang W, Wang YH, et al. Safety and efficacy of sofosbuvir-based direct-acting antiviral regimens for hepatitis $\mathrm{C}$ virus genotype 6 in Southwest China: real-world experience of a retrospective study. J Viral Hepat. 2019;26(3):316-322. doi:10.1111/jvh.13033

14. Hlaing NKT, Nangia G, Tun KT, et al. High sustained virologic response in genotypes 3 and 6 with generic NS5A inhibitor and sofosbuvir regimens in chronic $\mathrm{HCV}$ in myanmar. $J$ Viral Hepat. 2019;26(10):1186-1199. doi:10.1111/jvh.13133

15. Leroy V, Angus P, Bronowicki JP, et al. Daclatasvir, sofosbuvir, and ribavirin for hepatitis $\mathrm{C}$ virus genotype 3 and advanced liver disease: a randomized phase III study (ALLY-3+). Hepatology. 2016;63 (5):1430-1441. doi:10.1002/hep.28473

16. Blanco JR, Rivero-Juarez A. HCV genotype 3: a wolf in sheep's clothing. Expert Rev Anti Infect Ther. 2016;14(2):149-152. doi:10.1586/14787210.2016.1127757

17. Huang R, Rao H, Xie Q, et al. Comparison of the efficacy of sofosbuvir plus ribavirin in Chinese patients with genotype $3 \mathrm{a}$ or $3 \mathrm{~b}$ HCV infection. J Med Virol. 2019;91(7):1313-1318. doi:10.1002/ jmv. 25454

18. Cheinquer H, Sette H Jr, Wolff FH, et al. Treatment of chronic HCV infection with the new Direct Acting Antivirals (DAA): first report of a real world experience in Southern Brazil. Ann Hepatol. 2017;16 (5):727-733. doi:10.5604/01.3001.0010.2717

19. Hezode C, Lebray P, De Ledinghen V, et al. Daclatasvir plus sofosbuvir, with or without ribavirin, for hepatitis $\mathrm{C}$ virus genotype 3 in a French early access programme. Liver Int. 2017;37(9):1314-1324. doi:10.1111/liv.13383

20. Nelson DR, Cooper JN, Lalezari JP, et al. All-oral 12-week treatment with daclatasvir plus sofosbuvir in patients with hepatitis $\mathrm{C}$ virus genotype 3 infection: ALLY-3 phase III study. Hepatology. 2015;61 (4):1127-1135. doi:10.1002/hep. 27726

Infection and Drug Resistance

\section{Publish your work in this journal}

Infection and Drug Resistance is an international, peer-reviewed openaccess journal that focuses on the optimal treatment of infection (bacterial, fungal and viral) and the development and institution of preventive strategies to minimize the development and spread of resistance. The journal is specifically concerned with the epidemiology of
21. Belperio PS, Shahoumian TA, Loomis TP, et al. Real-world effectiveness of daclatasvir plus sofosbuvir and velpatasvir/sofosbuvir in hepatitis C genotype 2 and 3. J Hepatol. 2019;70(1):15-23. doi:10.1016/j.jhep.2018.09.018

22. Sarwar S, Tarique S, Aleem A, et al. Effect of adding daclatasvir in sofosbuvir-based therapy in genotype 3 hepatitis C: real-world experience in Pakistan. Eur J Gastroenterol Hepatol. 2019;31 (8):1035-1039. doi:10.1097/MEG.0000000000001376

23. Curry MP, O'Leary JG, Bzowej N, et al. Sofosbuvir and velpatasvir for $\mathrm{HCV}$ in patients with decompensated cirrhosis. $N$ Engl $J$ Med. 2015;373(27):2618-2628. doi:10.1056/NEJMoa1512614

24. El Serafy MA, Kassem AM, Omar H, et al. APRI test and hyaluronic acid as non-invasive diagnostic tools for post HCV liver fibrosis: systematic review and meta-analysis. Arab J Gastroenterol. 2017;18 (2):51-57. doi:10.1016/j.ajg.2017.05.005

25. Karic U, Pesic-Pavlovic I, Stevanovic G, et al. FIB-4 and APRI scores for predicting severe fibrosis in chronic hepatitis C a developing country's perspective in DAA era. J Infect Dev Ctries. 2018;12(3):178-182. doi:10.3855/jidc. 10190

26. Galle PR, Forner A, Llovet JM, et al. European association for the study of the liver. electronic address eee, clinical practice guidelines Panel C, representative EGB, Panel m. EASL recommendations on treatment of hepatitis C: final update of the series. J Hepatol. 2020;73 (5):1170-1218.

27. Pecoraro V, Banzi R, Cariani E, et al. New direct-acting antivirals for the treatment of patients with hepatitis c virus infection: a systematic review of randomized controlled Trials. J Clin Exp Hepatol. 2019;9 (4):522-538. doi:10.1016/j.jceh.2018.07.004

28. Wei L, Lim SG, Xie Q, et al. Sofosbuvir-velpatasvir for treatment of chronic hepatitis $\mathrm{C}$ virus infection in Asia: a single-arm, open-label, Phase 3 trial. Lancet Gastroenterol Hepatol. 2019;4(2):127-134. doi:10.1016/S2468-1253(18)30343-1

29. Tao YC, Deng R, Wang ML, et al. Satisfactory virological response and fibrosis improvement of sofosbuvir-based regimens for Chinese patients with hepatitis $\mathrm{C}$ virus genotype 3 infection: results of a real-world cohort study. Virol J. 2018;15(1):150. doi:10.1186/ s12985-018-1066-8

30. Han Q, Fan X, Wang X, et al. High sustained virologic response rates of sofosbuvir-based regimens in Chinese patients with HCV genotype 3 a infection in a real-world setting. Virol J. 2019;16(1):74. doi:10.1186/s12985-019-1184-y

31. Fried MW, Shiffman ML, Reddy KR, et al. Peginterferon alfa-2a plus ribavirin for chronic hepatitis $\mathrm{C}$ virus infection. $N$ Engl $\mathrm{J}$ Med. 2002;347(13):975-982. doi:10.1056/NEJMoa020047

32. Rinaldi L, Perrella A, Guarino M, et al. Incidence and risk factors of early $\mathrm{HCC}$ occurrence in $\mathrm{HCV}$ patients treated with direct acting antivirals: a prospective multicentre study. J Transl Med. 2019;17 (1):292. doi:10.1186/s12967-019-2033-x

33. Chen Yi Mei SLG, Thompson AJ, Christensen B, et al. Sustained virological response halts fibrosis progression: a long-term follow-up study of people with chronic hepatitis C infection. PLoS One. 2017;12(10):e0185609. doi:10.1371/journal.pone.0185609 antibiotic resistance and the mechanisms of resistance development and diffusion in both hospitals and the community. The manuscript management system is completely online and includes a very quick and fair peerreview system, which is all easy to use. Visit http://www.dovepress.com/ testimonials.php to read real quotes from published authors. 